University of Wollongong

Research Online

Faculty of Engineering and Information

Faculty of Engineering and Information

Sciences - Papers: Part A

Sciences

$1-1-2015$

Piezoelectric poly(vinylidene fluoride) microstructure and poling state in active tissue engineering

Clarisse Ribeiro

University of Minho

Daniela M. Correia

University of Minho

S Ribeiro

University of Minho

Vitor Sencadas

University of Wollongong, victors@uow.edu.au

Gabriela Botelho

University of Minho

See next page for additional authors

Follow this and additional works at: https://ro.uow.edu.au/eispapers

Part of the Engineering Commons, and the Science and Technology Studies Commons

Research Online is the open access institutional repository for the University of Wollongong. For further information contact the UOW Library: research-pubs@uow.edu.au 


\title{
Piezoelectric poly(vinylidene fluoride) microstructure and poling state in active tissue engineering
}

\begin{abstract}
Tissue engineering strategies rely on suitable membranes and scaffolds, providing the necessary physicochemical stimuli to specific cells. This review summarizes the main results on piezoelectric polymers, in particular poly(vinylidene fluoride), for muscle and bone cell culture. Further, the relevance of polymer microstructure and surface charge on cell response is demonstrated. Together with the necessary biochemical cues, the proper design of piezoelectric polymers can open the way to novel and more reliable tissue engineering strategies for cells in which electromechanical stimuli are present in their environment.
\end{abstract}

\section{Keywords}

tissue, active, state, poling, microstructure, engineering, fluoride, piezoelectric, vinylidene, poly

\section{Disciplines}

Engineering | Science and Technology Studies

\section{Publication Details}

Ribeiro, C., Correia, D. M., Ribeiro, S., Sencadas, V., Botelho, G. \& Lanceros-Méndez, S. (2015). Piezoelectric poly(vinylidene fluoride) microstructure and poling state in active tissue engineering. Engineering in Life Sciences, 15 351-356.

\section{Authors}

Clarisse Ribeiro, Daniela M. Correia, S Ribeiro, Vitor Sencadas, Gabriela Botelho, and Senentxu LancerosMéndez 


\section{Relevance of microstructure and poling state of piezoelectric poly(vinylidene fluoride) for active tissue engineering strategies}

Clarisse Ribeiro, ${ }^{1,}$, Daniela M. Correia ${ }^{1,3 \#}$, Sylvie Ribeiro ${ }^{1}$, Vítor Sencadas ${ }^{1,}{ }^{4}$, Gabriela Botelho $^{3}$, Senentxu Lanceros-Méndez ${ }^{1,2}$

${ }^{1}$ Centro/Departamento de Física, Universidade do Minho, Campus de Gualtar, 4710057 Braga, Portugal

2 INL - International Iberian Nanotechnology Laboratory, 4715-330 Braga, Portugal

${ }^{3}$ Centro/Departamento de Química, Universidade do Minho, Campus de Gualtar, 4710057 Braga, Portugal

${ }^{4}$ Escola Superior de Tecnologia, Instituto Politécnico do Cávado e do Ave, Campus do IPCA, 4750-810, Barcelos, Portugal

\# These authors contributed equally to this work.

\section{Corresponding author:}

Clarisse Ribeiro and Senentxu Lanceros-Méndez, Tel: +351253604320, Fax: +351253604061, email: cribeiro@fisica.uminho.pt and lanceros@ fisica.uminho.pt

Keywords: Piezoelectric polymer, PVDF, surface topography, physical stimuli, cell behavior 


\begin{abstract}
Tissue engineering strategies rely on suitable membranes and scaffolds providing the necessary physico-chemical stimuli to specific cells. This paper summarizes the main results on piezoelectric polymers, in particular poly(vinylidene fluoride), for muscle and bone cell culture. Further, the relevance of polymer microstructure and surface charge on cell response is demonstrated. It is thus shown that, together with the necessary biochemical clues, the proper design of piezoelectric polymers can open the way to novel and more reliable tissue engineering strategies for cells in which electromechanical stimuli are present in their environment.
\end{abstract}

\title{
Introduction
}

Materials used as scaffolds for tissue engineering applications are designed to match structural, morphological, mechanical and chemical properties of the tissue or organ that will be replaced. Apart from blood, most, if not all, human cells in human tissues are anchorage dependent, being therefore a critical issue the microstructure and surface properties of the scaffolds. This important issue is related to the extracellular matrix (ECM), which depends on the tissue under consideration, presenting multiple components and tissue-specific interactions [1]. Further, the understanding of the electrical properties of tissues and cells is attracting increasing attention. Electrical signals are present in many functions of living cells, i.e., they generate electromotive force, increase or decrease the differences in electrical voltage whenever necessary, use varying resistances in series or in parallel, switch on and off, control and rectify current flow and store charge [2-3]. An electrical voltage exists across the plasma membrane, with the inside of the cell remaining more negative than the outside. By convention, the potential outside the cell is zero; therefore, the typical value of the membrane potential is in the range of -60 to $-100 \mathrm{mV}[4]$.

Conductive polymers have been thus used for tissue engineering applications [3], but some drawbacks exists concerning these materials such as the need of an external power source to promote electrical stimuli to the cells.

An interesting possibility for applying electrical signals to biological tissues is the use of piezoelectric materials and, in particular, piezoelectric polymers. Piezoelectric polymers are materials that generate varying surface charges under mechanical solicitation and do not require additional energy sources or electrodes [5] for the 
generaltion of the electrical signal. These materials are thus suitable for novel active tissue engineering strategies in which electroactive response and scaffold microstructure will play an essential role.

\section{Poly(vinylidene fluoride) as piezoelectric polymer}

PVDF is a polymer with remarkable polymorphism, showing at least four crystalline phases: $\alpha, \beta, \gamma$ and $\delta$, being the $\beta$-phase the one with the highest piezoelectric coefficient [6-7] and consequently the most desirable for applications were the electrical response is needed.

The non-polar $\alpha$-phase is typically obtained from the melt at moderate or fast cooling rates, while $\beta$-PVDF is achieved by stretching films in the $\alpha$-phase at high stretching ratios at temperatures between 80 and $100{ }^{\circ} \mathrm{C}$. During the $\alpha$ to $\beta$-phase transformation, the spherulitic structure with a TGTG' (trans-gauche-trans-gauche) conformation characteristic of the $\alpha$-phase is destroyed and an all trans-planar "zigzag" microfibrillar polymer chain reorganization is obtained (figure 1a). This process results in films mostly in the $\beta$-phase, but with a small percentage of $\alpha$-phase material $[5,8-9]$. Unoriented films exclusively in the $\beta$-phase can be obtained from the crystallization of PVDF from solvent casting when solvent is removed at temperatures below $70{ }^{\circ} \mathrm{C}$. These films show a high degree of porosity [8]. Further, the $\beta$-phase of PVDF can be also nucleated by the inclusion of specific fillers such as ferrite nanoparticles, zeolites or ferroelectric particles, among others [6].

Electrospray and electrospinning revealed to be a versatile method to produce PVDF particles and fibers, respectively, due to its simplicity, cost-effectiveness and potential for scale-up [10]. PVDF particles obtained from electrospray present a mean diameter between $800 \mathrm{~nm}$ up to $6 \mu \mathrm{m}$, depending of the processing conditions, in particular on the polymer solution viscosity (figure 1c) [11-12]. When solution viscosity is high enough to promote polymer entanglement, PVDF random or aligned fibers can be obtained with average fiber diameters between $130 \mathrm{~nm}$ and $1.3 \mu \mathrm{m}$ (figure 1d) [13]. In these processes, PVDF crystallizes mainly in the $\beta$-phase (between $70-85 \%$ ) but a small amount of $\alpha-P V D F$ can be detected. During electrospray and electrospinning, solvent evaporation occurs at room temperature, which favors polymer crystallization in the electroactive phase $[11,13]$. 
When the electroactive $\beta$-PVDF is obtained is obtained from a stretching process the degree of crystallinity is typically around 40\% [9]. When solvent evaporation occurs at temperature below $70{ }^{\circ} \mathrm{C}$, the degree of crystallinity present in the porous membranes is around 55\%, which is also the degree crystallinity degree found for the polymer particles and fibers $[11,14]$. It is to notice that both degree of crystallinity and $\beta$-phase content are critical in determining the piezoelectric response of PVDF [9].

In order to enhance the piezoelectric response of $\beta$-PVDF, it is necessary to apply an electrical poling procedure both to to the films obtained by solvent casting and to the ones obtained after mechanical stretching [9]. On the other hand, electrospinning and electrospray techniques allows polymer electrical poling during fiber and particle processing [15] and therefore, suitable piezoelectric response.
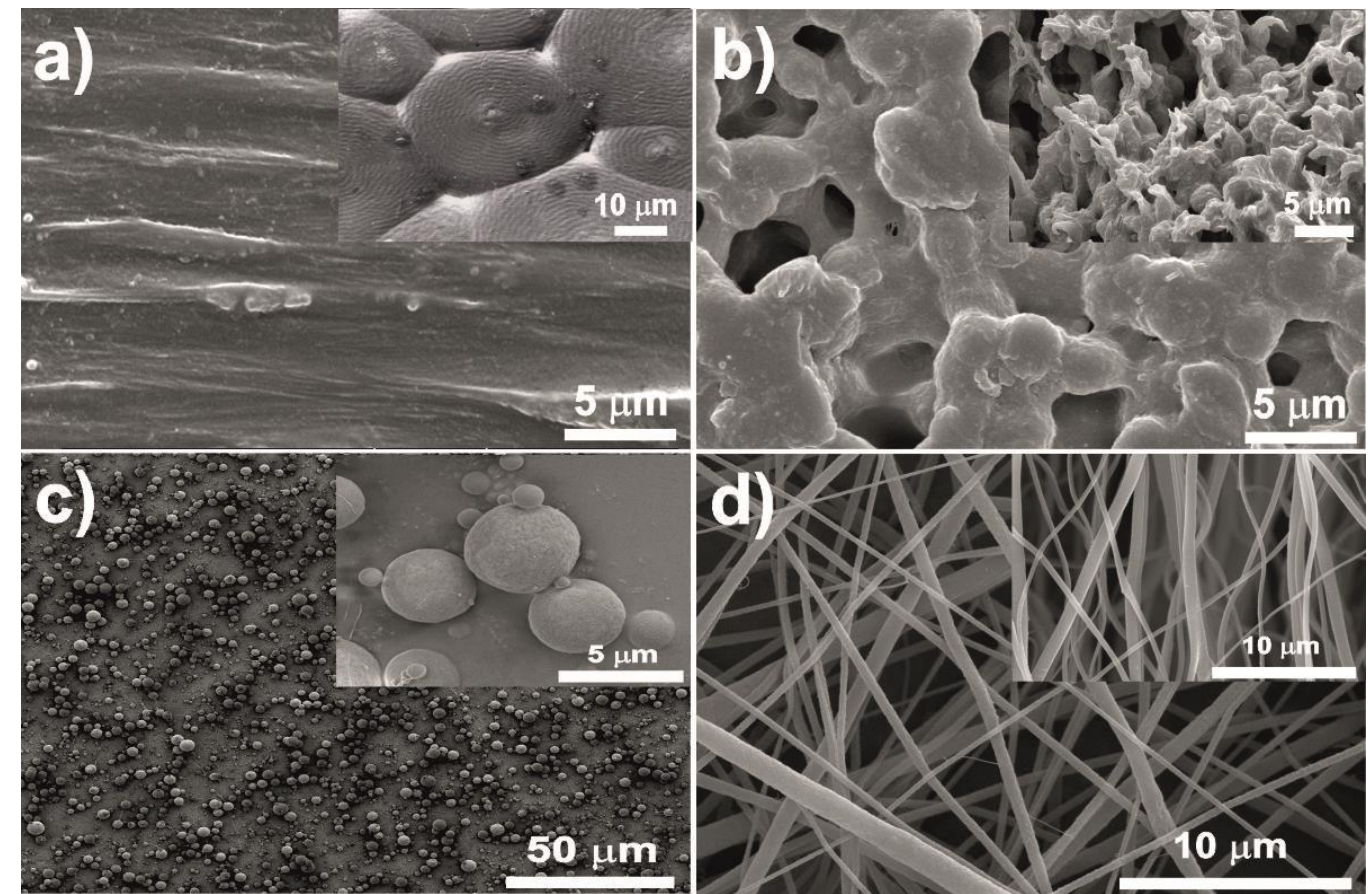

Figure 1 - Piezoelectric PVDF in the $\beta$-phase obtained with different morphologies: a) $\beta$-PVDF film obtained by stretching $(\mathrm{R}=5)$ at $80^{\circ} \mathrm{C}$ (inset: $\alpha$-PVDF spherulite), b) $\beta$ PVDF porous membrane obtained by solvent evaporation at room temperature, c) polymer microparticles obtained by electrospray and d) randomly electrospun polymer fibers obtained by electrospinning (inset: oriented polymer fibers).

\section{Surface properties and cell adhesion}

Cell/biomaterial interface plays an important role in cell adhesion and response. The behavior of different cell types on a given biomaterial shows different cell responses 
depending on cells intrinsic characteristics [16-18]. It is proven that different cell types use different attachment mechanisms for the same biomaterial surface and that also the same cell type can respond differently to different morphologies of the same biomaterial. When the cells adhere to the biomaterial surface, a sequence of chemical and physical reactions occurs between the cells and the biomaterial that influence the extracellular matrix deposition, cell proliferation and differentiation [18]. So, cell adhesion to the biomaterial surface involves a complex process and is affected by several parameters such as biomaterial surface chemistry [19-20], wettability [21-22], surface charge [23-24] and surface topography [17, 21, 25]. In this way, the main issue in the application and development of tissue engineering scaffolds is to understand the main factors influencing the cell adhesive ability. In this report, it is should be noted that only the effect of surface topography and poling state of the piezoelectric material will be focused.

The effect of the influence of biomaterial surface topography on cell response has been frequently addressed, but there is still a lack of comparable studies and results once there is no consensus for the topography characterization, using roughness parameters at different levels $[19,26]$. Once the cells are highly sensitive to their surrounding and respond to the environmental features at different scale levels, it is important to suitably define the cell environment [27-28].

In the process of cell adhesion, the cells attach to the biomaterials or scaffolds via local adhesion points that connect the cytoskeleton of the cell to the biomaterial surface. This interaction between the cells and the biomaterial is identified as focal adhesion. In this way, the focal adhesions are the predominant mechanism by which cells mechanically connect to and apply traction forces on their extracellular matrix, playing a critical role in cell response on biomaterials [29]. The cell adhesion usually involves extensive shape reorganization: the cell flattening ate the micrometer level and the enlargement of the initial contact areas at the submicrometer level [30]. Thus, the focal adhesion of the cells will sense their surrounding matrix and will transduce that information into morphological changes.

\section{Influence of PVDF microstructures on cell morphology}

Depending on the processing conditions and techniques, it is possible obtain PVDF samples with different topographies, surface poling states and different hydrophobicity (table 1) that will influence the cell response. 
It is important to note that the natural tissue exhibits a hierarchical structure, with specific properties at the macro-, micro- and nano-scale that affect significantly their behavior and function. In the same way, biomaterials/scaffolds show generally complex structures organized also at several length scales [31]. More specifically, the macroscopic level of the scaffold will provide guides and boundaries for cell growth and the micro- and nano-scale of the scaffold will provide the framework and capillary networks for the local cell growth and organization, allowing the cell adhesion and proliferation [32].

In this way, the design of bioactive membranes or scaffolds can be optimized by an understanding of the cell behavior cultured on these membranes/scaffolds. So, as previously mentioned, the topography design of the scaffold will influence the cell behavior and more specifically the cell morphology. Thus, it is essential for specific cells to select the suitable designs of the scaffold to induce appropriate cell response [33]. Additionally, the use of electric fields is beneficial or even necessary to stimulate specific cell types [34]. Piezoelectric polymers show in this way large potential for tissue engineering applications once electrical signals can be detected in human body and can be referred as a universal property of living tissue [35-36]. Thus piezoelectric PVDF samples can be tailored both with respect to morphology and electroactive response depending on the intended applications, including bone, muscle and neuronal tissue engineering applications (table 1).

Table 1 - Contact angle values (mean \pm SD) of the different PVDF samples depending on microstructure and poling state and their potential applications in tissue engineering. Poled + and - means poled samples with positive and negative surface charge, respectively.

\begin{tabular}{|c|c|c|}
\hline PVDF samples & $\begin{array}{l}\text { Contact angle } \\
\text { values }[23,24]\end{array}$ & $\begin{array}{c}\text { Applications in Tissue } \\
\text { Engineering }\end{array}$ \\
\hline Non-poled $\beta$-PVDF films & $83.1^{\circ} \pm 2.2^{\circ}$ & $\checkmark$ Bone $[23,37-39]$ \\
\hline "Poled +" $\beta$-PVDF films & $51.3^{\circ} \pm 3.1^{\circ}$ & $\checkmark$ Muscle [24, 40-41] \\
\hline "Poled -" $\beta$-PVDF films & $45.0^{\circ} \pm 1.6^{\circ}$ & $\checkmark$ Cartilage $[21,42]$ \\
\hline$\beta$-PVDF porous membranes & $94.3^{\circ} \pm 2.6^{\circ}$ & $\checkmark$ Neuronal [43-46] \\
\hline Aligned $\beta$-PVDF fibers & $135.1^{\circ} \pm 3.0^{\circ}$ & $\checkmark$ Spinal cord injury [47] \\
\hline Random $\beta$-PVDF fibers & $115.6^{\circ} \pm 3.3^{\circ}$ & $\checkmark$ Wound healing [48-49] \\
\hline
\end{tabular}


As specific examples of tissue engineering applications based on piezoelectric polymers, studies on muscle and bone regeneration will be presented in the following, as they are two examples of tissues particularly affected by electromechanical solicitations in their in vivo environment.

Skeletal muscle is a highly adapted tissue established by long parallel bundles of multinucleated myotubes that are formed by differentiation and fusion of myoblast. It has been demonstrated that PVDF substrates influence differently the morphology of C2C12 depending on its poling sate and morphology. It was observed that "poled -" $\beta$ PVDF films (figure 2a) induced higher proliferation than non-poled and "poled +" PVDF films. Here "poled -“ and "poled +" indicating poled samples with negative and positive surface charges on the cell culture side, respectively. However, the cells show an irregular morphology and random arrangement instead the elongated morphology of the skeletal muscle tissue [24]. Similar behavior was also observed in $\beta$-PVDF porous membranes and microparticles (figure $2 \mathrm{~b}$ and $\mathrm{c}$ ). In relation electrospun $\beta$-PVDF fibers, aligned PVDF fibers promoted the elongation of $\mathrm{C} 2 \mathrm{C} 12$ cells along the polymer fibers (figure 2d), contrarily to the randomly aligned PVDF fibers [24]. 

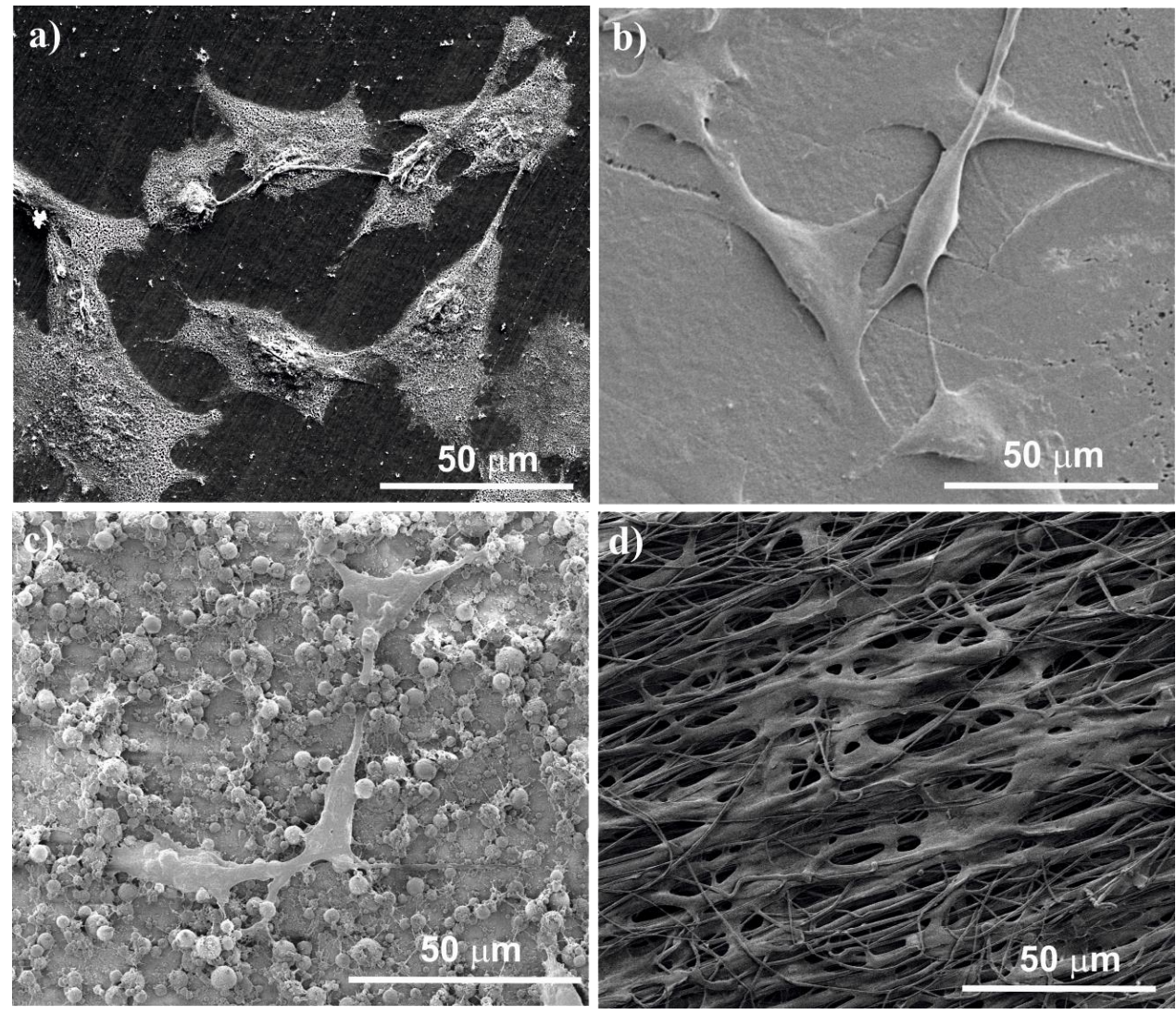

Figure 2 - Cell morphology of C2C12 myoblasts cells seeded on t PVDF samples with different microstructures after 1 day of cell culture: a) "poled -" $\beta$-PVDF film, b) $\beta$ PVDF porous membrane, c) electrospray polymer $\beta$-PVDF microparticles and d) $\beta$ PVDF aligned electrospun fibers.

In this way, it is shown that the combination of negatively surface charge with fiber orientation of PVDF scaffolds can provide suitably and necessary stimuli for proper muscle tissue engineering strategies.

Piezoelectric materials are particularly suitable for bone tissue engineering applications, since it was already demonstrated the piezoelectric behavior of bone [50]. It has been shown that different topographies and/or surface charge of PVDF samples can influence differently the behavior of bone cells. Contrary to the effects observed for muscle cells, higher adhesion and proliferation of MC3T3-E1 pre-osteoblast was observed in "poled +" $\beta$-PVDF samples [23, 51]. However, with human adipose stem cells (hASCs), "poled -" $\beta$-PVDF exhibited significantly larger total focal adhesion than non-poled and "poled +" samples, and consequently an improved cell differentiation [52]. These results indicate, as mentioned above, that cell adhesion is improved by the charged surfaces. 
Further, as in a piezoelectric material the electrical response is modulated by mechanical stimulation, experiments were performed with both types of cells under dynamic conditions, i.e. under varying mechanical solicitation. This mechanical stimulus is relevant in order to simulate the real environment of cell development, as bone formation in vivo is typically achieved under mechanical dynamic conditions. It was verified higher proliferation [23] and differentiation under dynamic conditions in poled $\beta$-PVDF samples than in static conditions. This behavior was not observed, on the other hand, for non-poled $\beta$-PVDF samples, indicating that varying surface charge, in addition to the topography, can support osteoblast cells differentiation and growth, the the piezoelectric scaffolds providing the necessary electrical stimuli for novel tissue engineering applications of this specific cells. Moreover, the previous works also demonstrate the relevance of developing suitable bioreactors mimicking different stimuli that can be found in the human body, providing specific physical signal for the improvement of the bone tissue engineering strategies.

\section{Conclusions}

This paper summarizes the main results on the use of piezoelectric poly(vinylidene fluoride) for muscle and bone cell culture. The relevance of tailoring both polymer microstructure and poling state for specific tissue regeneration is demonstrated by the different myoblast and osteoblast cell responses to similar polymer samples. It is thus shown that piezoelectric polymers allow proper design to develop novel and efficient tissue engineering strategies for cells needing electromechanical stimuli for their suitable development.

\section{Acknowledgements}

This work is funded by FEDER funds through the "Programa Operacional Fatores de Competitividade - COMPETE" and by national funds arranged by FCT- Fundação para a Ciência e a Tecnologia, project references PEST-C/FIS/UI607/2011 and PESTC/QUI/UIO686/2013. The authors also thank funding from Matepro -Optimizing Materials and Processes", ref. NORTE-07-0124-FEDER-000037", co-funded by the "Programa Operacional Regional do Norte" (ON.2 - O Novo Norte), under the "Quadro de Referência Estratégico Nacional" (QREN), through the "Fundo Europeu de 
Desenvolvimento Regional" (FEDER). The authors also thank support from the COST Action MP1003, 2010 'European Scientific Network for Artificial Muscles', MP1206 "Electrospun Nano-fibres for bio inspired composite materials and innovative industrial applications" and MP1301 "New Generation Biomimetic and Customized Implants for Bone Engineering". CR, DMC and VS thank the FCT for the SFRH/BPD/90870/2012, SFRH/BD/82411/2011 and SFRH/BPD/64958/2009 grants, respectively. Authors thanks the colleagues Andreia C. Gomes, José L. Gómez-Ribelles, F. Miguel Gama and Vesa P. Hytönen for intensive and pleasant collaboration and discussion in these issues.

The authors have declared no conflict of interest 


\section{References}

[1] Chan, B.P., Leong, K.W., Scaffolding in tissue engineering: general approaches and tissue-specific considerations. Eur Spine J 2008,17, 467-479.

[2] Kitchen, S. Electrotherapy: Evidence-based Practice. Edinburgh: Churchill Livingstone 2002.

[3] Ghasemi-Mobarakeh, L., Prabhakaran, M. P., Morshed, M., Nasr-Esfahani, M.H. et al., Application of conductive polymers, scaffolds and electrical stimulation for nerve tissue engineering. J. Tissue Eng. Regen. Med. 2011, 5, e17-e35.

[4] Matthews, G. G. Cellular Physiology of Nerve and Muscle. Malden, USA: Blackwell Publishing 2003.

[5] Lovinger, A. J. Developments in semicrystalline polymers. London: Elsevier Applied Sciences 1982.

[6] Martins, P., Lopes, A. C., Lanceros-Mendez, S., Electroactive phases of poly(vinylidene fluoride): Determination, processing and applications. Prog. Polym. Sci. 2014, 39, 683-706.

[7] Sencadas, V., Gregorio, R., Lanceros-Méndez, S., $\alpha$ to $\beta$ Phase Transformation and Microestructural Changes of PVDF Films Induced by Uniaxial Stretch. J. Macromol. Sci. B 2009, 48, 514-525.

[8] Sencadas, V., Gregorio, Jr R., Lanceros-Mendez, S., Processing and characterization of a novel nonporous poly(vinilidene fluoride) films in the $\beta$ phase. J. Non Cryst. Solids 2006, 352, 2226-2229.

[9] Gomes, J.; Nunes, J. Serrado; Sencadas, V.; et al., 
Influence of the beta-phase content and degree of crystallinity on the piezo- and ferroelectric properties of poly(vinylidene fluoride). Smart Materials \& Structures2010, $19,065010$.

[10] Zamani, M., Prabhakaran, M. P., Ramakrishna, S., Advances in drug delivery via electrospun and electrosprayed nanomaterials. Int. J. Nanomedicine 2013, 8, 29973017.

[11] Correia, D. M., Golcalves, R., Ribeiro, C., Sencadas et al., Electrosprayed poly(vinylidene fluoride) microspheres for tissue engineering applications. RSC Adv. 2014, 4, 33013-33021.

[12] Nasir, M., Matsumoto, H., Danno, T., Minagawa, M. et al., Control of diameter, morphology, and structure of PVDF nanofiber fabricated by electrospray deposition. $J$. Polym. Sci. B Polym. Phys. 2006, 44, 779-786.

[13] Ribeiro, C., Sencadas, V., Gómez-Ribelles, J. L., Lanceros-Méndez, S., Influence of processing conditions on polymorphism and nanofiber morphology of electroactive poly(vinylidene fluoride) electrospun membranes. Soft Mater. 2010, 8, 274-287.

[14] Na, H., Liu, X., Li, J., Zhao, Y. et al., Formation of core/shell ultrafine fibers of PVDF/PC by electrospinning via introduction of PMMA or BTEAC. Polymer 2009, 50, 6340-6349.

[15] Sencadas, V., Ribeiro, C., Bdikin, I. K., Kholkin, A. L. et al.,. Local piezoelectric response of single poly(vinylidene fluoride) electrospun fibers. Physica Status Solidi A Appl. Res. 2012, 209, 2605-2609. 
[16] Vladkova, T.G., Surface engineered polymeric biomaterials with improved biocontact properties. Int. J. Polym. Sci. 2010,DOI: 10.1155/2010/296094.

[17] Huaga, H. S., Choub, S. H., Don, T. M., Lai, W. C. et al., Formation of microporous poly(hydroxybutyric acid) membranes for culture of osteoblast and fibroblast. Polym. Adv. Technol. 2009, 20, 1082-1090.

[18] Chang, H-I., Wang, Y., Cell Responses to Surface and Architecture of Tissue Engineering Scaffolds 2011, DOI: 10.5772/21983.

[19] Anselme, K., Ploux, L., Ponche, A., Cell/material interfaces: influence of surface chemistry and surface topography on cell adhesion. J. Adhes. Sci. Technol. 2010, 24, 831-852.

[20] Brodbeck, W. G., Shive, M. S., Colton, E., Nakayama, Y. et al., Influence of biomaterial surface chemistry on the apoptosis of adherent cells. J. Biomed. Mater. Res. 2001, 55, 661-668.

[21] Areias, A. C., Ribeiro, C., Sencadas, V., Garcia-Giralt, N. et al., Influence of crystallinity and fiber orientation on hydrophobicity and biological response of poly(1lactide) electrospun mats. Soft Matter 2012, 8, 5818-5825.

[22] van Wachem, P. B., Beugeling, T., Feijen, J., Bantjes, A. et al., Interaction of cultured human endothelial cells with polymeric surfaces of different wettabilities. Biomaterials 1985, 6, 403-408.

[23] Ribeiro, C., Moreira, S., Correia, V., Sencadas, V. et al., Enhanced proliferation of pre-osteoblastic cells by dynamic piezoelectric stimulation. $R S C A d v .2012,2,11504-$ 11509.

[24] Ribeiro, S., Martins, P. M., Ribeiro, C., Sencadas, V. et al., Effect of poling state and morphology of piezoelectric poly(vinylidene fluoride) membranes for skeletal muscle tissue engineering. RSC $A d v$. 2013, 3, 17938-17944. 
[25] Kunzler, T. P., Drobek, T., Schuler, M., Spencer, N. D., Systematic study of osteoblast and fibroblast response to roughness by means of surface-morphology gradients. Biomaterials 2007, 28, 2175-2182.

[26] Macdonald, W., Campbell, P., Fisher, J., Wennerberg, A., Variation in surface texture measurements. J. Biomed. Mater. Res. B Appl. Biomater. 2004, 70B, 262-269.

[27] González-García, C., Sousa, S. R., Moratal, D., Rico, P. et al., Effect of nanoscale topography on fibronectin adsorption, focal adhesion size and matrix organisation. Colloids Surf. B Biointerfaces 2010, 77, 181-190.

[28] Martin, J. Y., Schwartz, Z., Hummert, T. W., Schraub, D. M. et al. Effect of titanium surface roughness on proliferation, differentiation, and protein synthesis of human osteoblast-like cells (MG63). J. Biomed. Mater. Res. 1995, 29, 389-401.

[29] Elineni, K. K., Gallant, N. D., Regulation of cell adhesion strength by peripheral focal adhesion distribution. Biophys. J. 2011, 101, 2903-2911.

[30] Pierres, A., Benoliel, A. M., Bongrand, P., Cell fitting to adhesive surfaces: A prerequisite to firm attachment andsubsequent events. Eur. Cell. Mater. 2002, 3, 31-45.

[31] Barthelat, F., Rim, J. E., Espinosa, H. D., A review on the structure and mechanical properties of mollusk shells - perspectives on synthetic biomimetic materials. In: Bhushan B, Fuchs H, editors. Applied Scanning Probe Methods XIII: Springer Berlin Heidelberg 2009, 17-44.

[32] Saltzman, W. M., Olbricht, W. L., Building drug delivery into tissue engineering design. Nat Rev Drug Discov 2002, 1, 177-186.

[33] Shoichet, M. S., Polymer Scaffolds for Biomaterials Applications. Macromolecules 2010, 43, 581-591.

[34] Lee, Y-S., Arinzeh, T. L., Electrospun nanofibrous materials for neural tissue engineering. polymers. Polymers 2011, 3, 413-426. 
[35] Foulds, I. S., Barker, A.T., Human-skin battery potentials and their possible role in wound-healing. Br J Dermatol 1983, 109, 515-522.

[36] Shamos, M. H., Lavine L.S., Piezoelectricity as a fundamental property of biological tissues. Nature 1967, 213, 267.

[37] Rodrigues, M. T., Gomes, M. E., Mano, J. F., Reis, R. L., beta-PVDF membranes induce cellular proliferation and differentiation in static and dynamic conditions. Adv. Mater. Forum 2008, 587, 72-76.

[38] Damaraju, S. M., Wu, S., Jaffe, M., Arinzeh, T. L., Structural changes in PVDF fibers due to electrospinning and its effect on biological function. Biomed. Mater. 2013, $8,045007$.

[39] Gimenes, R., Zaghete, M. A., Bertolini, M., Varela, J. A. et al., Composites PVDFTrFE/BT used as bioactive membranes for enhancing bone regeneration. Smart Mater. Struct. 2004, 5385, 539-547.

[40] Jansen, P. L., Klinge, U., Anurov, M., Titkova, S. et al., Surgical mesh as a scaffold for tissue regeneration in the esophagus. Eur Surg Res 2004, 36, 104-111.

[41] Inui, A., Kokubu, T., Makino, T., Nagura, I. et al., Potency of double-layered poly L-lactic acid scaffold in tissue engineering of tendon tissue. Int Orthop 2010, 34, 13271332.

[42] Ye, C., Hu, P., Ma, M-X., Xiang, Y. et al.,. PHB/PHBHHx scaffolds and human adipose-derived stem cells for cartilage tissue engineering. Biomaterials 2009, 30, 4401-4406.

[43] Young, T-H., Chang, H-H., Lin, D-J., Cheng, L-P., Surface modification of microporous PVDF membranes for neuron culture. J. Memb. Sci. 2010, 350, 32-41. 
[44] Valentini, R. F., Vargo, T. G., Gardella, Jr J. A., Aebischer, P., Electrically charged polymeric substrates enhance nerve fibre outgrowth in vitro. Biomaterials 1992, 13, 183-190.

[45] Valentini, R. F., Vargo, T. G., Gardella, J. A., Aebischer, P., Patterned neuronal attachment and outgrowth on surface modified, electrically charged fluoropolymer substrates. J. Biomater. Sci. Polym. Ed. 1994, 5, 13-36.

[46] Lee, Y. S., Collins, G., Livingston Arinzeh, T., Neurite extension of primary neurons on electrospun piezoelectric scaffolds. Acta Biomater. 2011, 7, 3877-3886.

[47] Ribeiro-Samy, S., Silva, N. A., Correlo, V. M., Fraga, J. S. et al., Development and characterization of a PHB-HV-based 3D scaffold for a tissue engineering and celltherapy combinatorial approach for spinal cord injury regeneration. Macromol. Biosci. 2013, 13, 1576-1592.

[48] Rouabhia, M., Park, H., Meng, S. Y., Derbali, H. et al., Electrical stimulation promotes wound healing by enhancing dermal fibroblast activity and promoting myofibroblast transdifferentiation. PLoS One 2013, 8, 1.

[49] Weber, N., Lee, Y. S., Shanmugasundaram, S., Jaffe, M. et al. Characterization and in vitro cytocompatibility of piezoelectric electrospun scaffolds. Acta Biomater. 2010, $6,3550-3556$.

[50] Fukada, E., Yasuda, I., On the piezoelectric effect of bone. J. Phys. Soc. Jpn. 1957, $12,1158-1162$.

[51] Ribeiro, C., Panadero, J. A., Sencadas, V., Lanceros-Méndez, S. et al., Fibronectin adsorption and cell response on electroactive poly(vinylidene fluoride) films. Biomed. Mater. 2012, 7, 035004.

[52] Pärssinen, J., Hammarén, H., Rahikainen, R., Sencadas, V. et al. Enhancement of adhesion and promotion of osteogenic differentiation of human adipose stem cells by 
poled electroactive poly(vinylidene fluoride). J. Biomed. Mater. Res. A 2014, doi: 10.1002/jbm.a.35234. 